\title{
La réception de l'école autrichienne en Italie
}

Die Rezeption der österreichischen Schule in Italien

The reception of the Austrian School in Italy

\section{Antonio Magliulo}

\section{OpenEdition}

\section{Journals}

Édition électronique

URL : https://journals.openedition.org/austriaca/1037

DOI : 10.4000/austriaca.1037

ISSN : 2729-0603

\section{Éditeur}

Presses universitaires de Rouen et du Havre

\section{Édition imprimée}

Date de publication : 1 juin 2020

Pagination : 67-86

ISBN : 979-10-240-1492-0

ISSN : 0396-4590

\section{Référence électronique}

Antonio Magliulo, «La réception de l'école autrichienne en Italie», Austriaca [En ligne], 90 | 2020, mis en ligne le 01 juin 2021, consulté le 03 octobre 2022. URL : http://journals.openedition.org/austriaca/ 1037 ; DOI : https://doi.org/10.4000/austriaca.1037

Ce document a été généré automatiquement le 3 octobre 2022.

Tous droits réservés 


\title{
La réception de l'école autrichienne en Italie
}

\author{
Die Rezeption der österreichischen Schule in Italien \\ The reception of the Austrian School in Italy
}

Antonio Magliulo

\section{Introduction}

1 De nos jours, l'école autrichienne jouit d'une excellente réputation en Italie : les livres de Ludwig von Mises, de Friedrich von Hayek et d'autres économistes dits « autrichiens » sont publiés et commentés avec intérêt, à l'intérieur et à l'extérieur des cercles académiques. Il y a plusieurs décennies, la situation était différente, rares étant les économistes italiens s'intéressant à cette école. Comment expliquer le succès relatif aujourd'hui et l'échec hier?

2 Avant et après la seconde guerre mondiale, une crise des idées libérales, auxquelles cette école est liée, puis la relance de théories favorables au libre-échange sur les marchés impactèrent à la fois les conceptions de la crise et la redécouverte de l'école autrichienne - en Italie comme dans le monde. À la fin du xxe siècle, il en fut de même après la chute des économies planifiées de type soviétique. Il faut également considérer des raisons d'ordre théorique. Des motifs analytiques ont, au fil du temps, conduit les économistes italiens à accepter ou à refuser les propositions de l'école autrichienne. Expliquer leur succès aujourd'hui implique de comprendre leur rejet hier. Un récit global de la réception de l'école autrichienne en Italie, resté en grande partie non écrit, est indispensable. On compte sur ce sujet quelques articles seulement, sur des auteurs ou des périodes limitées.

3 La présente étude entend contribuer à cette analyse, des origines à nos jours, pour montrer également des traits caractéristiques pertinents de la culture économique italienne. Notre aperçu historique fournira aussi des éléments d'analyse sur des thèmes 
économiques (ainsi, les «cycles d'affaires » ou business cycles). On peut observer trois grandes vagues de réception :

- la première couvre la période entre 1871, année de parution des Grundsätze de Menger, et 1918, dernière année de la Grande Guerre, qui marqua la fin d'une époque ;

- la deuxième couvre l'entre-deux-guerres, période connue en Italie sous le nom des « années de haute pensée théorique ";

- la dernière couvre l'après la seconde guerre mondiale, jusqu'à nos jours en somme, qui a été marquée par la «mort et la résurrection » de la pensée de Hayek ${ }^{1}$.

\section{Première vague (1871-1918) : en Italie, le marginalisme ne fut pas une révolution}

De 1861 à 1918, l'Italie s'unifie et se construit ; on parle souvent de l'« Italie libérale » menant à son terme un long processus d'unification nationale avec le Risorgimento. Les Habsbourg étaient regardés comme des tyrans étrangers, dont l'empire occupait des terres italiennes et entravait l'unification nationale. Il n'en avait cependant pas toujours été ainsi dans le passé : Marie-Thérèse a régné à Vienne et à Milan de 1740 à 1780 en souveraine aimée et éclairée. En revanche, un siècle plus tard, et surtout à partir de 1848 et de la répression des révolutions nationales qui ont éclaté partout en Europe et dans l'empire, les Habsbourg ont perdu tout capital de sympathie.

5 L'Europe centrale présentait deux projets politiques alternatifs: les Habsbourg voulaient régir un empire multinational tandis qu'en Allemagne, on bâtissait un empire national. L'Italie suivit la voie tracée par l'Allemagne, au long de trois guerres d'indépendance contre l'Autriche. Le 3 février 1871, Rome fut déclarée capitale d'une Italie incluant Milan et Venise, mais pas encore le Trentin ni la Vénétie julienne, sous domination autrichienne : c'étaient les «terres irrédentes ». Le Reich de Bismarck et de l'empereur Guillaume devint alors le grand allié et l'inspirateur de l'Italie. Si, en 1882, l'Italie rejoignit la "Triple Alliance", c'était afin de consolider ses relations avec l'Allemagne (et se protéger de possibles menaces françaises), pas par esprit de réconciliation avec l'Autriche-Hongrie. Bismarck poussa l'Italie à accepter la coalition, mais, étant donné le caractère exclusivement défensif du traité d'alliance, l'Italie n'était pas tenue d'intervenir d'entrée de jeu dans la Grande Guerre à l'été 1914. Après un an de neutralité, elle changea de camp, rejoignit la « Triple Entente » ennemie des empires centraux et combattit de nouveau l'Autriche. La première guerre mondiale constitue en Italie la " quatrième » (et dernière) " guerre d'indépendance » : les terres " irrédentes » (Trentin et Vénétie julienne) furent libérées et annexées à la faveur des traités de paix en 1919 et de la fin de l'empire austro-hongrois qui marqua aussi la fin du Risorgimento ${ }^{2}$.

6 La bataille se menait également sur le plan des idées. En Europe centrale, le Methodenstreit opposait l'Autrichien Carl Menger, depuis Vienne, à l'Allemand Gustav von Schmoller, à Berlin. Leurs écoles respectives, autrichienne marginaliste et allemande historiciste, se confrontaient et, par exemple, si les premiers défendaient la liberté d'échanger sur les marchés, les seconds étaient plus interventionnistes, des socialistes étatistes universitaires, surnommés Kathedersozialisten.

7 L'école « autrichienne » d'économie représentait l'une des trois inspirations directrices de la révolution dite «marginaliste » du début des années 1870 - les deux autres étant inspirées du Britannique Stanley Jevons et du Français Léon Walras. Durant la période 
précédente, l'économie dite «classique» expliquait grosso modo le développement économique par une propension à l'échange sur des marchés libres et la distribution par la répartition entre propriétaires terriens, capitalistes et prolétaires. La valeur d'échange des biens était essentiellement déterminée par leur coût de production. Des auteurs comme Karl Heinrich Rau étaient proches de la tradition classique ${ }^{3}$.

Les marginalistes reconsidérèrent le rôle de l'utilité dite "marginale » et réinventèrent la théorie de la valeur, que des Italiens comme l'abbé Galiani avaient pressentie au $\mathrm{xVIII}^{\mathrm{e}}$ siècle. Alfred Marshall et Vilfredo Pareto, de la génération suivant immédiatement les trois pères de la "révolution marginaliste ", soulignèrent l'idée que la valeur dépend à la fois de l'utilité et du coût; ils s'écartaient des vues « autrichiennes » de Menger et de ses disciples, Eugen von Böhm-Bawerk et Friedrich von Wieser, pour qui la valeur dépendait entièrement de l'utilité marginale.

La première édition des Grundsätze de Menger fut traduite en italien et publiée dans le Giornale degli Economisti en 1906 et 1907 (puis en un volume en 1909). La deuxième édition, celle publiée par le fils de Menger à titre posthume en 1923, qui diffère de la première, parut en italien en 1925. Sans doute la véritable « révolution » appartient au texte d'origine: les vues de Menger et de ses disciples directs bouleversent la vision classique des marchés et décrivent l'économie comme un processus de «remontée de filière ", où la valeur du produit fini ${ }^{4}$ détermine les ordres supérieurs selon une échelle de moyens de production (« remontant » vers l'origine des intrants dans la chaîne de production) - et pas l'inverse (l'approche classique). Israel Kirzner, héritier de cette école, écrit :

[Dans l'école autrichienne des origines] les valeurs ne sont pas (comme dans l'économie de Marshall) regardées comme conjointement déterminées par des considérations subjectives (utilité) et objectives (soit le coût en termes physiques). Les valeurs sont considérées plutôt comme déterminées exclusivement par les actions des consommateurs, qui agissent dans un cadre donné de produits existants et/ou de possibilités de production. Le coût est tout simplement considéré (par Menger et notamment par Wieser, dont le nom a fini par être étroitement lié à cette idée) comme une utilité potentielle, volontairement sacrifiée par substitution (afin de se rendre possesseur d'une utilité qu'on lui préfère) 5 .

Les économistes italiens discutèrent abondamment les idées nouvelles. Dès les années 1870 , ils s'intéressèrent aux questions de méthode sur le rôle économique de l'État. Francesco Ferrara prit la tête d'une ligue des défenseurs du marché libre, et Luigi Luzzatti celle des interventionnistes. On n'accepta toutefois vraiment en Italie la révolution marginaliste en tant que telle, notamment sa version autrichienne, qu'à partir de 1889, suite à la publication des Principi di economia pura (Principes d'économie pure) de Maffeo Pantaleoni, que Piero Sraffa, qui allait devenir le spécialiste de l'économiste David Ricardo, devait surnommer « le prince des économistes italiens ${ }^{6}$ ».

La thèse de Pantaleoni est que le marginalisme généralise, d'une manière qui peut paraître inattendue, des théorèmes majeurs des auteurs classiques sur les coûts réels (de production notamment). On peut en effet, selon lui, exprimer ces théorèmes indifféremment en termes d'utilité ou de coût. Si la valeur d'échange d'un bien provient de l'utilité de la dernière dose disponible de ce bien (son degré final d'utilité, c'est la thèse autrichienne), sa quantité disponible dépend, quant à elle, de son coût de production. À la marge, coût et utilité devraient coïncider et, dit Pantaleoni, « en ce sens, le coût de production de chaque chose est surtout simplement une autre façon de désigner son degré final d'utilité ${ }^{7}$ ». Comme dans la synthèse dite "néoclassique » avancée 
par Marshall, ce coût est vu comme un sacrifice (ou une "peine ») que toute production engendre inévitablement. Il s'agit d'un coût de production « tout psychologique » qui se traduit par la souffrance du travailleur et l'abstinence du capitaliste ${ }^{8}$.

Au début $\mathrm{du} \mathrm{xx}^{\mathrm{e}}$ siècle, toute une nouvelle série de manuels d'économie parut en Italie, où les thèses de la révolution marginaliste furent présentées systématiquement pour la première fois. Par des voies différentes, les principaux économistes (citons Luigi Cossa, Emilio Nazani, Camillo Supino, Augusto Graziani, Ghino Valenti, Enrico Barone, Achille Loria, Riccardo Dalla Volta) s'approprièrent les vues de Pantaleoni, à savoir que le coût de production est la somme totale de la souffrance du travailleur, et de « l'attente » du capitaliste (waiting). Le facteur coût est à la fois dans l'équilibre général selon Barone et dans les analyses de (courte, moyenne ou longue) période chez Valenti, Supino et Graziani.

Avec une telle vision du marginalisme, l'analyse de l'équilibre doit occuper le devant de la scène, sous la double version partielle «à la Marshall » ou générale «à la Pareto ». Pantaleoni regardait les deux approches comme complémentaires plutôt qu'alternatives. Au début, Pareto lui-même semblait accepter l'idée qu'une approche fondée sur des équilibres partiels devait échouer à capturer l'essence des phénomènes économiques, en réalité leur interdépendance concomitante sur les marchés. Pareto allait réfuter ensuite fermement cette vue qui fonde la synthèse de Pantaleoni.

Dans cette perspective, la formation de la théorie néoclassique de l'équilibre et de la croissance en Italie inspira deux stratégies alternatives et rivales de politique économique, que les économistes ont recommandées et parfois mises en pratique. Selon la première, l'État ne devait pas s'ingérer dans le fonctionnement spontané des marchés. Ses interprètes principaux étaient Pantaleoni lui-même, Pareto, Antonio De Viti de Marco et Luigi Einaudi. La politique économique alternative voyait dans l'État le garant des limites fixées dans un régime de prix flexibles et de taux de change stables. L'État pourrait (et devrait) mener une politique économique active afin de réconcilier les objectifs de croissance et de stabilité nationales. Les interprètes les plus influents de ce courant de pensée étaient Barone, Rodolfo Benini et Marco Fanno.

En suivant, au moins temporairement, l'approche de Pantaleoni, les principaux économistes italiens ont considéré le marginalisme comme une formule nouvelle intégrant et élargissant les notions économiques de l'école classique. C'était évidemment passer à côté de la révolution scientifique à l'œuvre, qu'ils n'ont simplement pas vue. En conséquence, la tendance fut de n'évaluer et de ne retenir des analyses autrichiennes que la contribution à la théorie de la valeur marchande, en refusant l'idée de supprimer le facteur «coût réel » de l'analyse. En 1916, lorsque s'achève cette première vague de réception de l'école autrichienne, Dalla Volta publie son ouvrage sur la théorie de la valeur dans ce sens et, à propos des économistes autrichiens, il écrit cette phrase concise et révélatrice de la profondeur du malentendu : «C'est une grave erreur de croire que l'économie est régie seulement par l'utilité ${ }^{9}$ ». 


\section{Deuxième vague de réception, dans les années 1930 : entre Hayek et Keynes}

16

le Grand Conseil fasciste appelle à voter sa destitution. Entre ces deux dates ${ }^{10}$, l'ascension et le déclin du chef fasciste ont marqué vingt ans qui firent de l'Italie un État totalitaire tentant de bâtir une économie planifiée dite " corporative ». Le terme indiquait l'engagement dans une "troisième voie » entre communisme et libéralisme, deux systèmes honnis, pour une société où les droits de propriété seraient garantis, mais où le marché resterait sous contrôle intégral de l'état (au moins en théorie, puisque la doctrine fasciste affirme explicitement que rien ne doit échapper à celuici) ${ }^{11}$.

Après 1929, en Italie comme ailleurs, le débat économique fut dominé par les questions de cycles économiques et les causes et les effets de la Grande Dépression. Ce débat était non seulement le lieu d'une bataille de géants, Hayek et Keynes, mais aussi des efforts alternatifs vers une "voie médiane ", que souhaitait l'économiste ordolibéral allemand Wilhelm Röpke. La réception de l'économie autrichienne en Italie coïncidait alors pour beaucoup avec une réflexion théorique constante sur le travail et la pensée de Hayek en comparaison des positions antagoniques de Keynes, d'une part, et de celles, proches, de Röpke, d'autre part. Il serait cependant peu utile de tenter d'évaluer l'influence de ces idées en Italie par le nombre et la qualité des revues et des traductions d'ouvrages d'économie: en fait peu de titres de ces auteurs furent publiés alors. Indiquons seulement, qu'en 1935, un essai de Hayek et un texte de Mises furent inclus dans le volume VIII de la prestigieuse et influente Nuova collana degli economisti (« Nouvelle collection des économistes »), avec une introduction de Fanno, alors que Francesco Vito et Costantino Bresciani Turroni publiaient des recensions d'œuvres de Hayek dans des revues internationales ${ }^{12}$. Hayek sur la théorie du surinvestissement dans les cycles d'affaires (business cycles), mais ils n'ont en général pas suivi son soutien du « laisser-faire » en politique. C'est la clef pour saisir l'attitude «italienne » à l'égard de Hayek, l'économiste "autrichien » respecté dans la théorie et peu suivi dans la pratique. Une raison est, avant tout, que ces économistes croyaient les cycles économiques provoqués par nombre d'événements, et pas seulement par les perturbations monétaires. Gustavo Del Vecchio et Francesco Vito invoquaient des facteurs réels (innovations technologiques) ou l'épargne forcée résultant de l'autofinancement des entreprises. Mauro Fasiani appliquait un principe fondé sur l'idée d'accélération, introduite par Albert Aftalion et Arthur Spiethoff. Giuseppe Ugo Papi s'appuyait sur la théorie de l'erreur de Wesley Mitchell, tandis que Guglielmo Masci observait le «baromètre » conçu par le Harvard Committee of Economic Research codirigé par Warren Parsons et Charles Bullock ${ }^{13}$.

Par ailleurs, ces économistes italiens décrivaient « à la Röpke », pour ainsi dire, la façon dont une récession "normale" (c'est-à-dire se maintenant dans les limites de fluctuations usuelles en régime capitaliste) pouvait dégénérer en une "grande dépression » dite " anormale », exigeant pour la surmonter qu'on prenne des mesures de type keynésien, soit une intervention active plutôt que des politiques " passives » du type que l'on estimait prôné par Hayek. Le débat était dominé en Italie par trois économistes: Einaudi, Bresciani Turroni et Fanno. À la même époque que Röpke, ils 
voyaient la crise de 1929 comme une récession due à l'excès d'investissement et de consommation et qui avait dégénéré en une dépression due à l'épargne excédentaire. Tous trois partageaient les idées néoclassiques sur le cycle économique et tenaient compte de l'analyse de Keynes relative au « point critique de la déflation ${ }^{14}$ ».

Enfin, ces mêmes économistes italiens restaient plutôt favorables à une politique économique plus active que celle se limitant à la monnaie "neutre " évoquée par Hayek. Einaudi et Bresciani Turroni se rapprochaient de Röpke en soutenant la nécessité d'interventions gouvernementales dites «conformes ». Ces actions exogènes au marché devaient protéger la véritable concurrence, mais aussi l'exercice de certains droits sociaux, ou nationaux. Avec Fanno, qui n'était certes pas un "économiste fasciste ", des intellectuels, eux favorables à bâtir l'économie " corporative ", purent de fait proposer un modèle de politique économique "conforme" (c'est-à-dire " cohérent » avec la théorie néoclassique de l'équilibre et de la croissance) et favorable à une action directe des pouvoirs publics sur le marché. Leur but était de garantir les objectifs néoclassiques (prix flexibles, taux de change stables et équilibre entre l'épargne et l'investissement), tout en pensant que les leçons de la crise et des changements structurels dans l'économie moderne impliquaient que le marché libre n'était plus capable d'assurer à lui seul les objectifs souhaités par les économistes libéraux - concernant l'économie de guerre, une autre question qui se posait alors, on affirma que les sociétés fiduciaires (c'est-à-dire les trusts et les cartels en plein essor) passaient après les intérêts nationaux.

21 La conviction était qu'on ne pouvait atteindre les objectifs visés que grâce à un secteur public « organique» formé par l'État total. Avec d'autres, Fanno définit les grandes lignes d'une politique organique pour une économie réglementée. L'objectif de prix flexibles et de taux de change stables devait être atteint par une politique de contrôle public direct sur les salaires, les prix, les cartels, voire, le cas échéant, les facteurs de la balance des paiements, tandis qu'on obtiendrait l'équilibre visé entre épargne et investissement par le contrôle public des banques et des grandes entreprises. En d'autres termes, le régime fasciste se donnait les mêmes objectifs que les auteurs néoclassiques et, s'il entendait utiliser pour ce faire des instruments similaires, c'était cependant de façon différente, adaptée à ce que ces économistes jugeaient être l'esprit des temps nouveaux.

En conséquence, ils optèrent pour une politique réduisant le secteur privé et relançant le secteur public. En novembre 1930, l'État italien, relayé par les syndicats fascistes, impose la réduction des salaires nominaux pour réduire le fardeau de la déflation. En parallèle, il finance des projets à long terme de "rénovation complète » des terres déjà cultivées et de drainage pour en rendre d'autres cultivables, soit des travaux publics énormes. En janvier 1933, on crée l'Institut de reconstruction industrielle (Istituto per la Ricostruzione Industriale), établissement public dont la mission est de racheter banques privées et entreprises au bord de la faillite pour lancer de vastes nationalisations. Le même mois, le Parlement approuve la loi obligeant les entreprises privées à requérir une autorisation à l'État pour toute construction d'usines nouvelles ou d'agrandissement de celles déjà existantes - l'objectif était d'obliger le secteur économique à financer un volume d'investissement qui ne devait en aucun cas dépasser l'épargne disponible.

23 Ces mesures provoquent une forte hausse du déficit public et aggravent le déséquilibre de la balance extérieure des paiements. En juillet 1933, l'Italie adhère au «bloc-or » qui 
réunit la France, la Belgique et d'autres pays, tous en quête de stabilité. En avril 1934, le gouvernement impose une nouvelle réduction des salaires, qui se révèle tout aussi inappropriée pour corriger le déséquilibre extérieur. En décembre, l'Italie suit les pas de la politique économique allemande en adoptant un contrôle gouvernemental total sur les taux de change. La mesure fut appliquée pendant un peu plus d'un an. En 1936, le «bloc-or » est anéanti et l'Italie doit également déprécier sa devise pour tenter de préserver la compétitivité de ses produits face à la concurrence internationale.

Les économistes italiens qui recensaient alors les ouvrages de Hayek sur les cycles économiques illustrent exemplairement ces contradictions. Attilio Cabiati et Francesco Vito (textes publiés en 1932 et 1934 respectivement) soulignaient qu'une politique de «neutralité de la monnaie » était incapable de garantir l'équilibre entre épargne et investissement. Dans une critique publiée par la revue Economica, Bresciani Turroni soutenait l'idée tirée de Monetary Theory and the Trade Cycle de Hayek, selon laquelle «le simple fait de changer le volume de la masse monétaire, même à niveau général des prix constant, suffit à "perturber la nature fermée du système économique" et à "l'éloigner de l'équilibre" 15 ». Bresciani Turroni disait aussi que la croissance économique de la fin des années 1920 ne pouvait pas durer, dès lors que les principales banques centrales, pour éviter la déflation, décidèrent de stabiliser le niveau des prix par une politique monétaire expansionniste. Même si l'épargne forcée, en termes hayékiens, pouvait impulser un mouvement cyclique dans l'économie, il ajoute qu'il existe en réalité des types variés d'épargne forcée, rendant la question plus complexe et impactant la réalisation de tout équilibre «vertical» dans la production. Bresciani Turroni écrit au final que « la situation est différente si l'épargne forcée est provoquée par l'impôt ou par les entreprises industrielles qui épargnent une partie de leurs profits plutôt que de le distribuer aux actionnaires ${ }^{16} »$.

Réfugié à Cambridge durant la période fasciste, Sraffa, qui devait promouvoir une théorie de l'échange de marchandises contre des marchandises dans son ouvrage publié en 1960, participait alors également au débat en critiquant radicalement l'idée d'une «monnaie neutre ${ }^{17} »$.

Ainsi, au cours des années 1930, les économistes italiens s'efforçaient de développer un modèle d'économie réglementée "corporatiste " en vue d'accomplir des objectifs de l'économie dite «libérale ». Ces économistes ont participé au débat international sur les cycles économiques et les causes et les effets de la Grande Dépression. S'ils se sentaient plus proches de Hayek, lorsqu'ils soutenaient que la source principale de l'instabilité conjoncturelle était la tendance à financer un volume d'investissement trop grand par rapport à l'épargne disponible, ils gardaient une position proche de Keynes pour interpréter la Grande Dépression comme due à une épargne excessive. Ils étaient souvent peut-être au fond plus proches des positions que Röpke mettait en avant, à savoir qu'un capitalisme moderne exigeait une politique néo-libérale d'intervention publique. 


\section{Troisième vague de réception des idées autrichiennes, après 1945 : quelle est la « route de la servitude », et comment l'éviter?}

Le bref récapitulatif des sections précédentes montre que la politique économique de l'Italie fut en somme en grande partie « conforme » à la pensée «néoclassique » - avec tout ce que ces termes devraient comporter de nuances et d'explications encore à apporter par l'histoire de la pensée économique. Le cadre restait une vision qui pouvait éventuellement en appeler à une politique de contrôle étatique des prix et des quantités.

Entre 1945 - ou mieux, après 1957, date du " Traité de Rome », une fois passé l'immédiat après-guerre - et 1992, c'est l'Europe qui a connu un essor extraordinaire. L'année 1992 marqua un tournant décisif dans l'histoire italienne avec la signature du Traité de Maastricht, d'une part, et l'enquête judiciaire "Mani pulite» ("Mains propres»), d'autre part, qui provoquèrent des chocs politiques forts sur le régime républicain établi après-guerre. C'est aussi au début des années 1990 qu'un processus de privatisation à grande échelle se mit en marche, qui allait aussi entraîner des changements profonds dans la vie économique. L'histoire économique italienne récente peut être divisée en ces deux sous-périodes, à savoir avant et après 1992.

La République italienne naît en juin 1946. Jusqu'en 1992, l'histoire des politiques économiques nationales se caractérise par une série d'essais pour orienter l'économie vers des objectifs (et des droits) sociaux protégés par la Constitution, à savoir sur l'emploi, l'instruction publique, l'aide sociale, etc. ${ }^{18}$ Après 1992, l'histoire est inversement marquée par une série de mesures prises au nom de ce que l'on appela une "révolution libérale ", visant à bâtir une économie "vraiment libre " grâce aux privatisations systématiques des biens publics et à la libéralisation des marchés. En Europe, et dans le reste du monde, ces changements radicaux débutent bien avant 1992. Il est de fait qu'avec bien des incompréhensions, le nom de Hayek leur servit parfois d'étendard et qu'il fut l'un des symboles de ladite révolution, comme si le héraut d'une révolution intellectuelle devait servir de porte-drapeau ou de nom de ralliement. En Italie, c'est ce qu'il faut analyser.

Hayek a publié en 1944 La route de la servitude, comme une mise en garde contre les effets du dirigisme, adressée aux « socialistes de tous les partis » auxquels l'ouvrage est dédicacé. Dans l'entre-deux-guerres, des pays d'Europe, et même la Grande-Bretagne avaient pu paraître abandonner, ou être tentés, d'abandonner le sentier de la liberté pour prendre, parfois presque par inadvertance, la voie fatale suivie en Allemagne. À l'approche de la fin de la guerre mondiale, l'ouvrage constituait un rappel de ce qu'il fallait à tout prix éviter : nous interprétons ici ce texte comme la thèse qu'il ne peut pas exister de troisième voie entre capitalisme et socialisme. Or le cas italien pourrait montrer qu'une troisième voie peut cependant ne pas nécessairement constituer une route vers la servitude. Quoi qu'il en soit, la réception de Hayek est parlante.

En 1947, Hayek cofonde la Société du Mont-Pèlerin, réunissant des partisans déterminés du libéralisme en provenance du monde entier. Parmi eux, Röpke fait figure, aux côtés de Hayek, de deuxième source d'inspiration possible ${ }^{19}$. Quelques années plus tard, ces deux grands esprits du néolibéralisme européen lancent dans cette Société un débat sur les instruments les plus efficaces pour défendre la concurrence contre les cartels et les monopoles. Les ordo-libéraux, menés par Röpke et 
par Walter Eucken, défendent un ordre constitutionnel avec une législation antitrust, alors que les "Autrichiens », menés par Hayek et Mises, restent favorables au libre jeu exclusif du marché et envisagent la légitimité de certains monopoles « spontanés ${ }^{20}$ ».

Avec La route de la servitude, Hayek eut sa (courte) période de gloire, suivie d'une longue «traversée du désert ». Il était marginalisé dans la communauté des économistes (au point que son recrutement fut refusé par le département d'économie de l'université de Chicago, dans laquelle il entra par le département des sciences sociales). Trente ans plus tard, en 1974, Hayek reçoit, de manière tout à fait inattendue, un prix Nobel d'économie pour sa contribution, datant des années 1930, à la théorie du cycle économique. À nouveau sur le devant de la scène, il retravaille sur les business-cycles et les causes des crises ${ }^{21}$. Dans les années 1970, il avait reconnu que la Grande Dépression avait constitué un cas particulier de situation exigeant le recours à des politiques expansionnistes de la part des gouvernements ${ }^{22}$.

La réception de l'économie autrichienne en Italie après la seconde guerre mondiale coïncide aussi avec une évaluation critique des ouvrages et de la pensée d'Hayek. Une fois passés de diverses positions adoptées sous le régime fasciste à de nouvelles positions dans la République italienne, les principaux économistes actualisèrent leurs ouvrages et les manuels d'économie qu'ils éditaient. En exagérant un peu, l'on pourrait presque dire que, souvent, il leur suffit pour cela de supprimer systématiquement l'adjectif "corporatif/corporatiste» avec un contenu et des objectifs quasiment inchangés. L'enquête portait de toute façon sur un modèle d'économie de marché réglementée. L'objectif demeurait souvent inchangé : trouver une "troisième voie " entre capitalisme et socialisme, par goût de la synthèse et rejet authentique de trancher pour une forme pure proposée par l'un des deux systèmes.

Einaudi et Bresciani Turroni convergeaient vers une "troisième voie» proche du néolibéralisme de Röpke en jugeant le libéralisme "à l'ancienne » inapte à protéger l'économie de marché. Sans politique économique active, le marché libre dégénérerait en régime monopolistique ou oligopolistique. Il fallait donc protéger la concurrence par des mesures « conformes » et, avant tout, une législation antitrust entravant cartels et monopoles. Le cas échéant, il fallait empêcher leur formation, ce dont la politique économique montra en pratique que ce ne fut pas le cas. En outre, le gouvernement devrait mieux prendre soin des populations dites "défavorisées » et "nécessiteuses ", par l'aide publique qu'il peut répandre. Le rôle de l'État va donc bien au-delà des limites établies par le marché, et cela dans le but de protéger ce dernier, mais aussi d'intégrer la population dans son jeu ${ }^{23}$.

La plupart des économistes italiens souhaitaient aller au-delà du néolibéralisme de Röpke, et bien loin des vues autrichiennes naturellement. Fanno et Vito, comme Pasquale Saraceno et Ezio Vanoni (entre autres) jugèrent qu'il n'était ni possible ni souhaitable de rétablir le type idéal d'un marché de concurrence pure et parfaite. Impossible, car des changements structurels profonds étaient survenus dans l'économie, et guère souhaitables, car le marché ne devait pas être une sorte de mécanisme suprême impersonnel décidant de la production ("disant quoi, où et comment produire»). Il fallait de grands objectifs politiques, comme maintenir le niveau d'emploi et un rythme de croissance tendant à l'égalisation entre Italie du Nord et Italie du Sud. À cet effet, les instruments politiques adéquats restaient de grands projets d'investissement, pour lesquels les fonds proviendraient de banques et des sociétés contrôlées par l'État, comme une agence mise en place après-guerre, l'Institut 
de reconstruction italien (IRI). La politique économique des gouvernements italiens de l'après-guerre suivit cette voie, activement axée sur l'offre et donc alignée sur les objectifs de la théorie néoclassique, mais avec une vision volontariste de croissance économique, très loin de la conception autrichienne de l'économie. En Italie, on regarde l'action de l'État comme indispensable à garantir des prix flexibles, des taux de change stables et un équilibre entre épargne et investissement. La réception des idées autrichiennes, celles de Hayek notamment, se lit dans l'Italie de l'après-guerre selon cette perspective qui lui est profondément contraire ${ }^{24}$.

Pourtant, ce serait une erreur de penser que les économistes italiens n'avaient rien compris du travail de Hayek, ou qu'ils lui avaient prêté peu d'attention. En avril 1947, le philosophe italien Carlo Antoni participait à la conférence inaugurale de la Société du Mont-Pèlerin et deux Italiens (Einaudi et Bresciani Turroni) étaient sur la liste des membres lue par Hayek. Certes les ouvrages majeurs de Hayek n'étaient pas encore traduits en italien, mais ils ne l'étaient pas plus dans d'autres langues, dont le français. Quant aux textes des origines, de Menger et de ses disciples de première génération, eux traduits (à la différence du français), ils remontaient à longtemps. Il y avait peu d'exceptions, mais il y en avait ${ }^{25}$ et il serait tout de même faux de dire que les économistes italiens n'avaient pas d'intérêt pour les idées de Hayek dès la deuxième moitié des années 1940.

D'ailleurs, les rares éditions françaises des travaux de Hayek jouèrent comme médiations par les critiques favorables qu'elles reçurent dans le Giornale degli economisti. Attilio Cabiati y dit dès 1940 son admiration pour L'économie dirigée en régime collectiviste. De l'ouvrage de Hayek, il partage l'idée qu'il est toujours risqué de légitimer une politique d'intervention gouvernementale, quelle qu'elle soit. Ferdinando Di Fenizio exprimait le même genre d'appréciation concernant La route de la servitude dans un texte de 1947 : il y constatait que Hayek, à la différence de Röpke, soulignait plus l'importance de ce que les gouvernements devraient s'abstenir de faire (en latin, les non agenda) plutôt que ce qu'ils devraient faire (agenda). La traduction italienne de La route de la servitude provoqua à son tour un grand émoi du fait de son retard même ${ }^{26}$. Pour finir, ce livre parut en mars 1948 chez l'éditeur Rizzoli sous le titre Verso la schiavitù (Vers la servitude).

Quant à un autre ouvrage de Hayek, Collectivist Economic Planning (L'économie dirigée en régime collectiviste), il est paru chez "Einaudi Editore» en mars 1946 sous le titre Pianificazione economica collettivista. L'avant-propos éclairant de Bresciani Turroni illustre les raisons pour lesquelles les économistes italiens (hormis les communistes) jugeaient qu'une société collectiviste qui abolirait la propriété privée serait intrinsèquement inapte à répondre aux besoins humains fondamentaux. Bresciani Turroni explique de manière convaincante comment le même résultat (insatisfaisant) adviendrait dans une société capitaliste où il n'existerait pas de marché libre pour les échanges, même si les droits de propriété étaient préservés. La question porte donc moins sur ces derniers que sur la logique des échanges. Pour autant, selon Bresciani Turroni, Hayek évitait le point fondamental, à savoir comment préserver la concurrence et éviter les monopoles.

Par la suite, des interprétations critiques variées des textes de Hayek virent le jour. En 1949, Antonio Pesenti, économiste membre du Parti communiste italien (PCI), publie un ouvrage au titre étonnant, La route de la servitude de Hoffman à Pella (La via della servitù ovvero da Hoffman a Pella), où il considère l'œuvre de Hayek comme « ouvrage de faible 
valeur scientifique, quoique d'une forte notoriété ", et il renvoie aux personnages de son titre, méconnus, mais qui gagneraient selon lui à être mieux connus. Dans le camp opposé, un ami de Hayek, Bruno Leoni, qui rejoindra la Société du Mont-Pèlerin en 1951, publie dès 1950 deux longues critiques favorables à Individualisme et ordre économique (Individualism and Economic Order) de Hayek et à L'Action humaine (Human Action) de Mises ${ }^{27}$. Dans un après-guerre compliqué en Italie, ni les options de Pesenti ni celles de Leoni n'évaluent objectivement l'impact des idées autrichiennes : il faut pour cela lire Francesco Vito.

En janvier 1949, Vito publie une recension de La route de la servitude à l'occasion d'un article de synthèse dans une série portant sur l'idée de "fédération européenne ». Il fait observer, en particulier à propos du dernier chapitre de Hayek (" The prospects of international order ») qu'une "Fédération » (sciemment écrite avec une majuscule), européenne ou plus largement internationale, n'est jamais qu'un moyen de limiter le pouvoir des États-nations à l'intérieur de leurs économies, un engagement contraignant, comme les liens qu'Ulysse s'est imposés pour ne pas succomber aux sirènes. Ici, il s'agissait d'éviter l'appel d'une "troisième voie» planiste. Or Vito désapprouve Hayek en cela, car il voit, quant à lui, dans la fédération un lien qui peut unir des États-nations. Vito voulait une fédération européenne gérée par un gouvernement supranational sur des principes (clairement d'inspiration catholique) de subsidiarité, solidarité et bien commun.

41 Vers 1950, le rideau tombe de toute manière sur le travail de Hayek qui avait suscité un intérêt seulement bref, en Italie comme ailleurs. La faible lumière qui continue de luire à la Société du Mont-Pèlerin attire quelques intellectuels italiens qui y trouvent refuge. Leoni fut une exception en s'insérant comme protagoniste dans le débat opposant Hayek et Röpke ${ }^{28}$. En dehors de cela, l'époque était favorable à d'autres courants.

C'est donc l'effet d'un coup de tonnerre qu'eut le prix Nobel de 1974 (plus exactement le «prix de la Banque de Suède en l'honneur d'Alfred Nobel»). Le projecteur fut à nouveau braqué sur Hayek, qui revint au cœur des débats. Il inspirait dans une certaine mesure les «révolutions libérales» conduites par Margaret Thatcher en GrandeBretagne et Ronald Reagan aux États-Unis. En Italie aussi, on s'intéressa de nouveau à lui, mais... dix ans plus tard. Les traductions jouèrent cette fois un rôle crucial. À partir de 1986, elles abondèrent, de sorte que l'on compte à présent des centaines d'articles et de livres sur Hayek et l'école "autrichienne ». Cette efflorescence tardive vit des universitaires de disciplines variées (re)découvrir ces œuvres et cette école. Pour Hayek, la «troisième voie " (prônant une intervention gouvernementale) était une «route de la servitude » : cela ne détournait pas la plupart des économistes italiens conseillers des politiques de l'État de continuer de la rechercher. Einaudi et Bresciani Turroni avaient favorisé une voie "à la Röpke»; Fanno, Vito, Saraceno, Vanoni et d'autres voulaient dépasser le néolibéralisme, mais il s'agissait toujours de promouvoir des objectifs plus sociaux que libéraux, et certes pas autrichiens.

\section{Épilogue}

L'étude de la transmission, de l'assimilation et de l'adaptation des idées économiques d'une école donnée dans une nation fait mieux saisir l'esprit de sa culture économique ${ }^{29}$. Une identité de perspective fondamentale lie l'économie autrichienne et l'approche philosophique subjectiviste. L'économie autrichienne voit dans le marché le 
seul lieu des échanges et dans ses rouages les seuls mécanismes permettant aux consommateurs d'exprimer librement leurs préférences (personnelles et subjectives). Les prix y sont des signaux que les consommateurs envoient aux entreprises, qui essaient de les saisir au mieux, et chaque entrepreneur veut avant ses concurrents produire pour satisfaire ces besoins au plus tôt et au mieux. Le marché est le mécanisme de découverte de l'inconnu; il est donc par construction en avance sur la réglementation qui lui est même contradictoire, car aucun législateur ne peut deviner avec assurance quel genre ni quel nombre d'entreprises serviraient idéalement tel marché particulier. Moins qu'aucun autre, une autorité antitrust ou un gouvernement ne peuvent savoir ce que seul le marché se charge mécaniquement de sélectionner spontanément.

Les économistes italiens conçurent souvent le marché comme lieu où des préférences subjectives confluent tandis que des obstacles objectifs gênent les producteurs. Les prix sont le résultat de ces deux forces convergentes, et le marché doit être réglementé et géré par des actes d'intervention " conformes ». Les économistes italiens ont porté une certaine attention aux idées de l'économie autrichienne, en n'en acceptant cependant qu'une partie. Ils considéraient le subjectivisme autrichien uniquement comme une contribution à l'explication de la valeur marchande et la description apportée par Hayek de l'expansion provoquée par un surinvestissement seulement comme une contribution destinée à la compréhension des cycles économiques. Mais ils étaient persuadés que la valeur et les cycles peuvent être expliqués grâce à la théorie néoclassique. La « valeur » est bien plus qu'une simple utilité, alors qu'une politique de « monnaie neutre » ne suffit pas à garantir l'équilibre macroéconomique.

Le dialogue intellectuel avec l'école autrichienne a fait sortir deux caractéristiques significatives de la tradition économique italienne. La première est la propension à "élargir » la synthèse néoclassique plutôt qu'à l'abandonner. Pantaleoni a essayé d'abord de réconcilier Ricardo et Menger, et ensuite Marshall et Pareto ; de la même manière, Bresciani Turroni, Einaudi et Fanno ont essayé de réconcilier Hayek et Keynes. La deuxième caractéristique est leur préférence pour un type d'intervention gouvernementale "conforme" à la théorie néoclassique. En Italie, le libéralisme économique comme le Keynésianisme a souvent été une ligne de réflexion minoritaire.

Enfin, par rapport aux économistes autrichiens, il ressort un gène dominant de l'héritage italien : une recherche constante de la troisième voie entre le capitalisme et le socialisme. Les économistes italiens ont appris et apprécié la leçon autrichienne sur les dangers pour la liberté qui découle du socialisme (la deuxième voie) et de la planification aussi (la troisième voie). Mais ils ont pu également entrevoir les risques inhérents au capitalisme (la première voie) qui, plutôt que la "route de la liberté ", pouvait devenir une «route de la servitude». En effet, comme l'ont montré Bresciani Turroni et Einaudi, sans des formes d'intervention "conformes", le libre marché pouvait dégénérer en un régime oligopolistique et monopolistique. Certains économistes italiens (Vito et d'autres) sont allés au-delà et ont mis en évidence le risque pour les personnes de devenir esclaves d'une idole, le risque que le marché soit considéré comme un mécanisme suprême et impersonnel ayant le pouvoir de déterminer quoi, comment et où produire : ils souhaitaient utiliser le marché et non en devenir esclaves. 


\section{BIBLIOGRAPHIE}

N.B. Les titres ci-dessous s'ajoutent aux références figurant dans les notes.

BRESCIANI TURRONI Costantino, « Prefazione », dans Friedrich A. von Hayek et al., Pianificazione economica collettivistica, Torino, Einaudi, 1946, p. XI-XXI.

CABIATI Attilio, « La moneta "neutrale" in un libro del Dr. Hayek », La Riforma sociale, nº 2, 1932, p. 194-204.

CABIATI Attilio, « A proposito di un symposium sul sindacalismo », Giornale degli economisti e Annali di economia, $\mathrm{n}^{\circ}$ 5-6, 1940, p. 373-380.

DI FENIZIO Ferdinando, « La "Via della servitù" di F. A. v. Hayek », Giornale degli economisti e Annali di economia, $\mathrm{n}^{\circ} 1-2,1947$, p. 31-42.

HAYEK Friedrich A., The Road to Serfdom, London, Routledge, 1944.

INFANTINO Lorenzo, « Postfazione », dans Friedrich A. von Hayek, Autobiografia, Soveria Mannelli, Rubbettino, 2011, p. 215-230.

LEONI Bruno, La sovranità del consumatore, Roma, Ideazione, 1997.

MENGER Carl, Grundsätze der Volkswirtschaftslehre, Wien, Wilhelm Braumüller, 1871 [trad. italienne, Imola, $1909 ; 2^{e}$ éd., Wien, 1923, trad. italienne, Principii fondamentali di economia politica, Bari, Laterza, 1925 ; nouv. éd., Principî di economia politica, Raimondo Cubeddu (dir.), Soveria Mannelli, Rubbettino, 2001.

MIROWSKI Philip et PLEHWE Dieter (dir.), The Road from Mont Pelerin. The Making of the Neoliberal Thought Collective, Cambridge, Harvard University Press, 2009.

PESENTI Antonio, « La via della servitù ovvero da Hoffman a Pella », Critica economica, n 3 , 1949, p. 29-57.

VITo Francesco, « Luci ed ombre del federalismo europeo », Rivista internazionale di scienze sociali, $\mathrm{n}^{\circ} 1,1949$, p. $47-55$.

\section{NOTES}

1. Concernant la diffusion des idées économiques en général, nous nous inspirons de Peter A. Hall (dir.), The Political Power of Economic Ideas: Keynesianism across Nations, Princeton, Princeton University Press, 1989, et de José L. Cardoso, "The International Diffusion of Economic Thought ", dans Warren J. Samuels, Jeff E. Biddle et John B. Davis (dir.), A Companion to the History of Economic Thought, Malden, Blackwell, 2003, p. 622-633. Sur l'économie autrichienne, voir Israel M. Kirzner, " Austrian School of Economics », dans John Eatwell, Murray Milgate et Peter Newman (dir.), New Palgrave. A Dictionary of Economics, London, Macmillan, 1987, p. 145-151; Eugen M. Schulak et Herbert Unterköfler, The Austrian School of Economics, Auburn, Ludwig von Mises Institute, 2011. Sur la pensée économique italienne, voir Riccardo Faucci, A History of Italian Economic Thought, London, Routledge, 2014.

2. Sur l'histoire politique italienne, voir notamment les travaux de Gilles Pécout, Naissance de l'Italie contemporaine (1770-1922), Paris, Nathan, 1997 ; sur l'histoire économique italienne, ceux de 
Gianni Toniolo, An Economic History of Liberal Italy, 1850-1918, London, Routledge, 2014, et de Vera Zamagni, An Economic History of Italy, 1860-1990, Oxford, Clarendon Press, 1993.

3. Sur ces écoles et les auteurs qui en étaient membres, voir Heinz D. Kurz « German and Austrian schools ", dans Gilbert Faccarello et Heinz D. Kurz (dir.), Handbook on the History of Economic Analysis, Vol. II: Schools of Thought in Economics, Cheltenham, Edward Elgar, 2016, p. 252-273.

4. Il s'agit des biens disponibles à la consommation, soit le dernier stade de la chaîne de production; ils sont rangés et numérotés comme les biens premiers, au sens où ils sont les plus proches de l'utilité finale du consommateur.

5. Israel Kirzner, op. cit., p. 146-147, italiques dans l'original, la traduction est la nôtre. Sur les auteurs de l'époque de Menger, notamment Rau, voir Gilles Campagnolo, Critiques de l'économie politique classique. Marx, Menger et l'École historique, Paris, Matériologiques, 2014 et Heinz D. Kurz, op. cit.

6. Le livre de Pantaleoni fut traduit en anglais en 1898, sous le titre Pure Economics. Sur la diffusion du marginalisme en Italie, voir Piero Barucci, "The spread of marginalism in Italy, 1871-1890 ", History of Political Economy, n 4, 1972, p. 512-532 ; Mauro Gallegati, « The spread of Alfred Marshall's economics in Italy, 1885-1925 », dans Rita McWilliams Tullberg (dir.), Alfred Marshall in Retrospect, Cheltenham, Edward Elgar, 1990, p.133-151; Vitantonio Gioia, "Gli economisti italiani e la "scuola austriaca": dalla teoria del valore alla scienza delle finanze ", dans Piero Barucci (dir.), Le frontiere dell'economia italiana. Gli economisti stranieri in Italia: dai mercantilisti a Keynes, Firenze, Polistampa, 2003, p. 325-351; Raimondo Cubeddu, «I liberisti nella cultura politica italiana ", Philosophy Kitchen, ${ }^{\circ}$ 8, mars 2018, p. 61-94.

7. Maffeo Pantaleoni, Principii di economia pura [1889], Milano, Treves, 1931, p. 232 (italiques de l'original).

8. Voir Maffeo Pantaleoni, "Prefazione", dans Carl Menger, Principii fondamentali di economia politica [1909], Bari, Laterza, 1925, p. XIII-XVI. Pour la réception de Menger en Italie, voir en particulier Flavia Monceri, « Sulla fortuna di Carl Menger in Italia », dans Carl Menger, Principî di economia politica, Raimondo Cubeddu (éd.), Soveria Mannelli, Rubbettino, 2001, p. 351-382.

9. Riccardo Dalla Volta, La teoria moderna del valore economico, Mantova, Mondovi, 1916, p. 127 : «Credere che tutta l'economia sia governata dalla utilità, e solamente da essa, è un grave errore » (notre traduction).

10. Parallèlement, l'empire austro-hongrois avait laissé place à une Autriche de taille très réduite et soumise à des vicissitudes non moins grandes, au point de disparaître en 1938. Le sort de l'« école autrichienne » n'est pas seulement lié à l'entité géographique qui s'évanouit alors, mais les principaux économistes autrichiens furent contraints à l'exil.

11. Sur l'économie italienne de l'entre-deux-guerres, voir Riccardo Faucci, op. cit., chap. 7. Sur le débat concernant les «cycles d'affaires » en économie et sur la Grande Dépression, voir Antonio Magliulo, "The Great Depression of 1929 in Italy. Economists' Views and Government Policy ", dans Michalis Psalidopoulos (dir.), The Great Depression in Europe: Economic Thought and Policy in a National Context, Athens, Alpha Bank Historical Archives, 2012, p. 153-185.

12. Voir Friedrich A. Hayek, «Una politica errata: il rialzo artificiale dei prezzi », La Borsa, $\mathrm{n}^{\circ} 25$, 1934, p. 3-4, et Le vicende della valuta aurea [1932], Torino, UTET, « Nuova Collana di Economisti », 1935, vol. 8, p. 523-537 ; Ludwig von Mises, La stabilizzazione del potere d'acquisto della moneta e la politica della congiuntura [1928], Torino, UTET, « Nuova Collana di Economisti », 1935, vol. 8, p. 23-90 ; Attilio Cabiati, «La moneta "neutrale" in un libro del Dr. Hayek », La Riforma sociale, $\mathrm{n}^{\circ}$ 2, 1932, p. 194-204; Costantino Bresciani Turroni, « Review of Monetary Theory and the Trade Cycle. By Friedrich A. Hayek, Jonathan Cape 1933 », Economica, août 1934, p. 344-347 ; Francesco Vito, « Recensione di F. A. von Hayek, Preise un Produktion and Monetary Theory and the Trade Cycles », Rivista internazionale di scienze sociali, $\mathrm{n}^{\circ}$ 1, 1934, p. 136-139.

13. L'instrument était constitué de trois courbes relatives à trois groupes de phénomènes (disons, trois «marchés ») : l'activité spéculative (A), la production (B), les phénomènes monétaires $(\mathrm{C})$. 
Ses concepteurs voulaient voir si et comment les variations de la courbe A précédaient celles de $B$, avant celles de la courbe $C$. Selon eux, en examinant le premier groupe de phénomènes, on devait pouvoir prévoir l'évolution du second, et ainsi de suite. Ce «baromètre » ne se révéla pas un outil de prévision efficace lors de la grande crise de 1929 ; il fut abandonné dès 1935.

14. Voir Antonio Magliulo, op. cit.

15. Costantino Bresciani Turroni, op. cit., p. 345.

16. Ibid., p. 346.

17. Voir Piero Sraffa, « Dr. Hayek on Money and Capital », Economic Journal, n ${ }^{\circ} 1,1932$, p. $42-53$ et "A Rejoinder », Economic Journal, n 2, 1932, p. 249-251. Voir aussi un commentateur proche des positions de Sraffa, Heinz D. Kurz, «Capital Theory, Crises, and Business Cycles: The Triangular Debate between Hayek, Keynes, and Sraffa », Journal of Reviews on Global Economics, $\mathrm{n}^{\circ} 4,2015$, p. 186-191.

18. Voir Riccardo Faucci, op. cit., chap. 8.

19. Voir Ronald M. Hartwell, A History of the Mont Pelerin Society, Indianapolis, Liberty Fund, 1995 ; Philip Mirowski et Dieter Plehwe (dir.), The Road from Mont Pélerin. The Making of the Neoliberal Thought Collective, Cambridge, Harvard University Press, 2009.

20. Cependant, en s'interrogeant sur l'interventionnisme des ordo-libéraux, Stefan Kolev observe: "Si Mises les mettait [les ordo-libéraux] dans la catégorie des "interventionnistes allemands", Hayek, dès les années 1930 et 1940, avait systématiquement cherché à se rapprocher d'Eucken, de Röpke et de leurs collègues, et il avait commencé à bâtir son économie politique et sa philosophie sociale sur des bases vraiment très proches des thèmes explorés par les ordolibéraux ", dans "Ludwig von Mises and the "Ordo-interventionists": more than just aggression and contempt? », CHOPE Working Paper, $\mathrm{n}^{\circ}$ 35, 2016 (notre traduction).

21. Voir Alan Ebenstein, Friedrich Hayek. A Biography, New York, Palgrave, 2001, et Daniel S. Jones, Masters of the Universe. Hayek, Friedman, and the Birth of Neoliberal Politics, Princeton, Princeton University Press, 2012.

22. Voir Antonio Magliulo, «Hayek and the Great Depression of 1929. Did he really change his mind? », European Journal of the History of Economic Thought, $\mathrm{n}^{\circ}$ 1, 2016, p. 31-58.

23. Voir Constantino Bresciani Turroni, Introduzione alla politica economica, Torino, Einaudi, 1942, et Il programma economico-sociale del liberalismo, Milano, Giuffrè, 1945 ; Luigi Einaudi, « Economia di concorrenza e capitalismo storico. La terza via fra i secoli XVIII e XIX », Rivista di storia economica, $\mathrm{n}^{\circ}$ 1, 1942, p. 49-72, et Lezioni di politica sociale, Torino, Einaudi, 1949.

24. Voir Sergio Noto, "Introduzione", dans Alan Ebenstein, Friedrich von Hayek. Una biografia, Soveria Mannelli, Rubbettino, 2009, p. 5-32.

25. Il est vrai que l'édition française de Collectivist Economic Planning (L'économie dirigée en régime collectiviste) avait été publiée en 1935 et que La route de la servitude, publiée en 1944, parut en français en 1945, alors que ces livres ne parurent en italien qu'en 1946 et 1948. Mais cela ne révèle pas forcément un manque d'intérêt ni de l'indifférence de la part des économistes italiens. Sur la réception de l'économie autrichienne en France, voir Gilles Campagnolo, « De Carl Menger à Karl Menger - à Charles Menger ? Sur la diffusion de la pensée économique autrichienne ", Austriaca, $\mathrm{n}^{\circ}$ 63, décembre 2006, p. 133-150.

26. Les motifs ont été narrés en détail par Lorenzo Infantino en 2011 : la correspondance privée de la maison d'édition Einaudi a révélé que ce retard venait de ce qu'une "très mauvaise traduction » avait été fournie par une jeune protégée du patriarche de la philosophie libérale italienne, le grand penseur Benedetto Croce. Il fallait tout reprendre.

27. Leoni réunit ces textes pour les rééditer en 1997.

28. Il fut actif dans l'accord connu sous le nom de « compromis d'Erhard » et, en 1960, il devint secrétaire de la Société. En 1961, il en organisa la $12^{\mathrm{e}}$ assemblée générale, à Turin (avec le soutien de Sergio Ricossa) et il en prit en 1967 la présidence. Voir Ronald M. Hartwell, op. cit., chap. 6. 
29. Et, comme les idées économiques passent les frontières nationales, ce type de recherche culturelle pourrait devenir un instrument puissant pour enquêter sur les communautés d'idées sur le plan trans- ou multinational, comme en Europe.

\section{RÉSUMÉS}

Cette étude analyse la réception de l'économie autrichienne en Italie, de ses débuts jusqu'à nos jours, en mettant en évidence, par des comparaisons, les caractéristiques pertinentes de la culture économique italienne. Un aperçu historique de la réception autrichienne et du keynésianisme montre comment les économistes italiens légitimèrent à la fois l'approche autrichienne et la politique keynésienne dans les crises anormalement graves. Une première vague va de 1871, année de parution des Grundsätze de Menger, à 1918, fin de la Grande Guerre et d'une époque où les économistes italiens, en privilégiant Alfred Marshall et Vilfredo Pareto, accueillirent le marginalisme pour intégrer théorie classique et idées autrichiennes. La deuxième vague (l'entre-deux-guerres), période dite de "haute théorie», vit des économistes majeurs s'aligner sur les vues de Hayek quant au surinvestissement. Une troisième vague mène de l'aprèsguerre à nos jours avec la « mort » et la « résurrection » de Hayek.

Dieser Artikel untersucht die Rezeption der Österreichischen Schule in Italien von 1871 bis heute und berücksichtigt dabei die Diskussion über den Konjunkturzyklus während der großen Depression der 1930er Jahre. Der Vergleich dieser Theorien hat das italienische Wirtschaftsdenken geprägt, besonders die Fähigkeit, sich dem Mainstream anzupassen und den österreichischen Marginalismus mit dem Keynesianismus zu vereinen. In diesem Prozess gibt es drei Perioden. In der ersten Periode (1889-1916) sahen die italienischen ökonomen im Marginalismus eine Methode, um die klassische Theorie und die österreichische Ökonomie als einen Beitrag zur Marktpreistheorie zu integrieren. Sie bevorzugten die Versionen von Alfred Marshall und Vilfredo Pareto. In der Zwischenkriegszeit (die Jahre einer „höheren Lehre“) teilten die führenden Ökonomen die Ansichten Hayeks über Überinvestitionen in Konjunkturzyklen, aber billigten auch Keynes Politik in einer extremen Krise. Die dritte Periode, von 1945 bis heute, lässt sich durch das Vergessen und die darauffolgende Wiederentdeckung der Theorien Hayeks kennzeichnen.

This study of the reception of the Austrian School in Italy starts from its original reception to our days, through a comparative study aiming to find relevant traits in Italian economic culture. Our historical view shall bring insights into the peculiar traits of Italian economics. One may distinguish three time periods: from the publication of Menger's Principles (1871) to 1918, end of World War I and of an era when Italian economists favored views by Alfred Marshall and Vilfredo Pareto. Between the wars (the years of so-called "high theory") Italian economists participated in debates over "business cycles" dominated by Hayek and Keynes. Features of Italian economics emerge through this comparison, as leading economists shared the overinvestment (Hayekian) theory of business cycles, yet justified Keynesian policies to tackle the abnormal Great Depression. After World War II and until our days, a third wave of reception saw the "decline and revival" of studies about Hayek. 
INDEX

Mots-clés : école autrichienne, culture économique, Italie

Keywords : austrian school, economic thought, Italy

Schlüsselwörter : österreichische schule, nationalökonomie, Italien, wirtschaftsgeschichte

\section{AUTEUR}

ANTONIO MAGLIULO

Università degli Studi internazionali di Roma, Rome 